\title{
Frecuencia de parásitos gastrointestinales en cuyes reproductoras de crianza intensiva
}

\author{
Frequency of gastrointestinal parasites in breeders guinea pigs of intensive breeding
}

\author{
Meylin Huamán ${ }^{1}$, Marjorie Killerby ${ }^{2}$, Lilia Chauca ${ }^{1}$
}

\section{RESUMEN}

El objetivo del estudio fue determinar la frecuencia de parásitos gastrointestinales presentes en cuyes reproductoras de crianza intensiva, así como el grado de infección parasitaria. Se recolectó 250 muestras de intestino delgado, intestino grueso y ciego, los cuales se colocaron en frascos de boca ancha, identificados y transportados a $4^{\circ} \mathrm{C}$. Las muestras fueron procesadas por las siguientes técnicas parasitológicas: directo, flotación y sedimentación. La identificación específica de parásitos en muestras positivas se realizó mediante un examen microscópico a través de características morfológicas de los huevos. Además, se determinó el grado de parasitismo de las infecciones. Del total de muestras analizadas el 37,2 $\pm 6,0 \%$ (93/250) resultaron positivos a parásitos gastrointestinales en reproductoras. Los parásitos identificados fueron Paraspidodera uncinata (20,4\%), Eimeria caviae (12,0\%), Capillaria sp. (4,8\%), Balantidium sp. (4,4\%), Trichuris sp. (2,0\%), Passalurus sp. (0,4\%) y Entamoeba sp. (0,4\%). El 79,6\% de las muestras positivas presentaron asociaciones monoparasitarias y el 20,4\% biparasitarias. El parasitismo mixto más frecuente fue $E$. caviae y $P$. uncinata. El grado de infección fue leve $(26.8 \%)$ para la mayoría de los animales positivos a $P$. uncinata, E. caviae, Capillaria sp, Balantidium sp, Trichuris sp, Passalurus sp y Entamoeba sp. Se concluye que P. uncinata y $E$. caviae son los parásitos más frecuentes encontrados en reproductoras de crianza intensiva. Se encontró asociación estadística entre el número de partos y el ambiente de crianza frente a la presentación de parasitosis.

PALABRAS CLAVE: Cuyes, parasitismo, crianza intensiva, Paraspidodera uncinata; Eimeria caviae.

\section{SUMMARY}

The objective of the study was to determine the frequency of gastrointestinal parasites present in intensively breeding reproductive guinea pigs, as well as the degree of parasitic infection. 250 samples of small intestine, large intestine and blind from guinea pigs were collected prior to the autopsy, which will be placed in wide-mouth bottles, identified and transported at $4{ }^{\circ} \mathrm{C}$. The samples were processed by the following parasitological techniques: direct, flotation and sedimentation. The specific identification of parasites in positive samples was performed by microscopic examination through the morphological characteristics of the eggs; In addition to determining the degree of parasitism of infections. Of the total samples analyzed, 37,2 $\pm 6,0 \%(93 / 250)$ were positive for gastrointestinal parasites in breeders. The parasites identified were Paraspidodera uncinata (20,4\%), Eimeria caviae (12,0\%), Capillaria sp. (4,8\%), Balantidium sp. (4,4\%), Trichuris sp. (2,0\%), Passalurus sp. (0,4\%) and Entamoeba sp. $(0,4 \%)$. The $79,6 \%$ of the positive samples associations of monoparasitic association and 20,4\% biparasitic. The most frequent mixed parasitism was E. caviae and $P$. uncinata. The degree of infection was mild (26,8\%) for the majority of animals positive for P. uncinata, E. caviae, Capillaria sp, Balantidium sp, Trichuris sp, Passalurus sp 
and Entamoeba sp. It is concluded that $P$. uncinata and E. caviae are the most frequent parasites found in intensively breeding breeders. Statistical association was found between the number of birth and the breeding environment versus the presentation of parasitosis.

KEYWORDS: Guinea pigs, parasitism, intensive breeding, Paraspidodera uncinata; Eimeria caviae.

\section{INTRODUCCIÓN}

La presentación de enfermedades parasitarias es una de las limitantes en la producción de cuyes, siendo estas responsables de más de las dos terceras partes de la morbilidad y mortalidad en esta especie (Instituto de Investigación Agraria (INIA), 1994; Florián, 1999). En la mayor parte de los casos, la infección parasitaria es gradual, los cuyes no presentan signos clínicos marcados y aparentemente se encuentran sanos. Las enfermedades parasitarias pasan desapercibidas por los criadores, producen retraso en el crecimiento y una mayor susceptibilidad para contraer otro tipo de enfermedades, traduciéndose en pérdidas económicas no cuantificables para los productores (Chauca, 1997; García, Chávez, Pinedo y Suárez, 2013).

Existen factores epidemiológicos específicos que contribuyen a la elevada presencia de ecto y endoparásitos en cuyes, como las deficientes condiciones higiénicas y sanitarias de las pozas, sobrepoblación animal, crianza con otras especies domésticas y ausencia de programas de prevención y control parasitario; estos factores son favorecidos por la baja condición socio económica y cultural del criador (INIA y CIID, 1991; INIA, 1994). El inadecuado manejo de la temperatura, humedad, corrientes de aire, densidad, limpieza en camas o dietas mal balanceadas pueden predisponer al animal a padecer de eventos de estrés que generen una inmunosupresión, incrementando la vulnerabilidad a diferentes enfermedades (Morales, 2013).

En el caso de los cuyes, usualmente las infecciones parasitarias son mixtas, es decir por varias especies parasitarias; cada una de las cuales ocupa un lugar determinado del tracto intestinal, produciendo transtornos nutricionales y fisiológicos variados (Florián, 2004). La gastroenteritis parasitaria esencialmente es una enfermedad de animales jóvenes, ya que los adultos desarrollan una respuesta relativamente resistente (Chauca, 1997; Florián, 2004).
Dentro de los protozoarios, Eimeria caviae es la especie parasitaria económicamente importante, los más susceptibles son los animales jóvenes después del destete (Vargas, Chávez, Pinedo, Morales y Suarez, 2014; Suárez, Morales y Villacaqui, 2014; Flausino et al., 2014; Becerra, 2015; Morales, 2017). Los nemátodos habituales de los cuyes son Paraspidodera uncinata, Trichuris $s p$, Capillaria $s p$ y Passalurus sp. (Murga, Terán y Cabanillas, 2000; Dittmar, 2002; Sánchez, 2013; Vargas et al., 2014; Suárez et al., 2014; Becerra, 2015; Morales, 2017). En el caso de las reproductoras, la prevalencia de los distintos endoparásitos es variada, P. uncinata (74\%), Capillaria spp. (34\%), Trichostrongylus sp. (6.1\%) y Trichuris spp. (3.4\%) (Ríos, 2018).

Actualmente, se ha logrado avances técnicos en la crianza comercial del cuy, pero los conocimientos sobre aspectos sanitarios y epidemiología de las enfermedades son aún escasos (Layme, Perales, Chavera, Gavidia y Calle, 2011). Los sistemas de producción intensivos de cuyes, consideran programas de control sanitario contra algunos patógenos bacterianos y ocasionalmente ectoparásitos; sin embargo, no se consideran medidas de control específicas contra los endoparásitos, debido a la forma de presentación de las infestaciones (Becerra, 2015).

En este contexto, el objetivo del estudio fue determinar la frecuencia de parásitos gastrointestinales presentes en cuyes reproductoras de crianza intensiva, así como el grado de infección parasitaria.

\section{METODOLOGÍA}

El estudio se realizó en los laboratorios de Sanidad del Programa de Cuyes del Instituto Nacional de Innovación Agraria (INIA) y el laboratorio de Parasitología de la Facultad de Medicina Veterinaria y Zootecnia de la Universidad Peruana Cayetano Heredia (FAVEZ-UPCH) Los especímenes procedían de granjas de crianza comercial de la provincia de Lima, de los distritos de La Molina y Pachacamac. Las muestras se recolectaron entre mayo de 2016 y mayo de 2019. 
El tamaño de muestra se determinó mediante la fórmula para estimar una proporción basada en la aproximación normal a la distribución binomial, con 95\% de confianza y 5\% de precisión (Daniel, 2005), considerando $80 \%$ de prevalencia previa (Sánchez, 2013) para infecciones parasitarias de crianza familiar comercial. Bajo estas condiciones, el tamaño muestral fue de 246 para el periodo determinado para el estudio (2016 a 2019).

Las muestras fueron recolectadas de cuyes hembras reproductoras que morían en el día, identificándose el número de partos y el ambiente de donde se encontraban (poza, jaula o parrilla). Los cuyes fueron llevados al laboratorio de sanidad de cuyes del INIA, para realizar la necropsia y tomar la muestra en el momento. Se recolectó muestras de intestino delgado, grueso y ciego, ligando cada extremo para evitar el derrame del contenido, y se colocaron en frascos de boca ancha. Las muestras fueron transportadas al Laboratorio de Parasitología de la FAVEZ - UPCH utilizando una caja térmica con bloques de gel refrigerante a temperatura de 3 a $4^{\circ} \mathrm{C}$. Entre la muerte de los animales y la recolección de las muestras no transcurrió más de 2 horas.

Las muestras de tracto gastrointestinal fueron procesadas utilizando la técnica directa (Beltrán, Tello y Náquira, 2003), la técnica de Sedimentación de Ritchie y la técnica de Flotación de Sheater (Rodríguez y Cob, 2005). El grado de parasitismo se evaluó de acuerdo a la cantidad de huevos por campo encontrados por flotación y sedimentación, se categorizó según 0,1 a 2, 3 a 5 y mayor a 6 huevos por campo, estas categorías se expresaron como negativo, leve, moderado y severo, respectivamente.
Los resultados fueron expresados mediante estadística descriptiva (frecuencia absoluta y relativa) para determinar el género de agentes parasitarios diagnosticados con mayor frecuencia. Los resultados fueron expresados en forma porcentual y el intervalo de confianza al $95 \%$ se calculó para el resultado general, los demás resultados mostraron la frecuencia para los diferentes estratos de las variables. Se utilizó la prueba de Ji Cuadrado para determinar la existencia de asociación entre el diagnóstico del parásito y las variables independientes: número de partos en las hembras reproductoras, estación y ambiente de crianza.

\section{RESULTADOS}

Del total de muestras analizadas, el 37,2 \pm $6,0 \%(93 / 250)$ resultaron positivos a endoparásitos (nemátodos y protozoos). Entre las muestras positivas se identificaron 7 géneros. Los nematodos hallados fueron: Paraspidodera uncinata, Trichuris spp., Capillaria sp., Passalurus sp. y como especies de protozoo: Eimeria caviae, Balantidium sp., y Entamoeba $s p$. El detalle de las frecuencias se encuentra en la tabla 1.

El 79,6\% de las muestras presentaron asociaciones monoparasitarias, siendo las especies más prevalentes P. uncinata $(38,7 \%)$ y E. caviae $(20,4 \%)$. Asimismo, se encontró $20,4 \%$ de biparasitismo, siendo la asociación más frecuente E. caviae y $P$. uncinata con $7,5 \%$. El detalle según especies se presenta en la tabla 2.

El grado de parasitismo evaluado en reproductoras fue negativo y leve para la mayoría de las especies parasitarias. El detalle se muestra en la tabla 3..

Tabla 1. Frecuencia de especies parasitarias en cuyes hembras reproductoras durante los años Mayo 2016 Mayo 2019

\begin{tabular}{lcc}
\hline Especies Parasitarias & Muestras positivas & Prevalencia \% e IC \\
\hline P. uncinata & 51 & 20,4 \\
E. caviae & 30 & 12,0 \\
Capillaria $s p$. & 12 & 4,8 \\
Balantidium $s p$ & 11 & 4.4 \\
Trichuris $s p$ & 5 & 2,0 \\
Passalurus $s p$. & 1 & 0,4 \\
Entamoeba $s p$. & 1 & 0,4 \\
Total de muestras & 250 & $37,2 \pm 6,0$ \\
\hline
\end{tabular}


En la tabla 4 se muestra los resultados de muestras positivas según estación, número de partos y ambiente de crianza. Para el caso de estación del año, del total de muestras estudiadas, se observó que en invierno el $44,2 \%$ de las reproductoras fueron positivas a alguna forma parasitaria. Aun así, no se encontró asociación entre el parasitismo y la estación. Respecto al número de partos, se observó mayor frecuencia en hembras de III y más de IV partos, $40,0 \%$ y $59,1 \%$, respectivamente. Para este caso sí se encontró asociación estadística significativa $(p<0,05)$. En cuanto al ambiente de crianza, se evidenció que los animales criados en poza mostraron $49,0 \%$ de positividad a alguna forma parasitaria, seguidos de los animales criados en jaulas; encontrándose asociación estadística significativa $(\mathrm{p}<0,05)$.

\section{DISCUSION}

Las enfermedades parasitarias en cuyes han sido objeto de numerosos reportes desde hace varios años, sin embargo, dichas investigaciones se limitan a cada tipo de crianza. En el este trabajo se encontró que el $37,2 \%$ de cuyes de crianza intensiva presentan parasitosis gastrointestinal, esta baja frecuencia podría estar relacionada al manejo de los animales en jaula o pozas con parrilla, que permiten tener menor grado de contaminación por formas infectivas (estadios larvarios). Los resultados se aproximan a lo reportado por Suárez et al., (2014) y Becerra (2015), que obtuvieron una prevalencia de 49,51\% y $43,1 \%$, en sistemas de crianza intensiva en Junín y en reproductoras en Moquegua, respectivamente.

Tabla 2. Asociaciones parasitarias encontradas en cuyes reproductoras de crianza intensiva, durante el periodo mayo 2016 - mayo 2019 ( $\mathrm{n}=93)$.

\begin{tabular}{lcc}
\hline ASOCIACIÓN & $\mathbf{n}$ & $\mathbf{\%}$ \\
\hline MONOPARASITISMO & 74 & 79,6 \\
P. uncinata & 36 & 38,7 \\
E. caviae & 19 & 20,4 \\
Balantidium sp. & 8 & 8,6 \\
Capillaria sp. & 7 & 7,5 \\
Trichuris $s p$ & 3 & 3,2 \\
Entamoeba sp. & 1 & 1,1 \\
BIPARASITISMO & 19 & 20.4 \\
E. caviae + P. uncinata & 7 & 7,5 \\
P. uncinata + Capillaria sp. & 5 & 5,4 \\
E. caviae + Balantidium sp. & 3 & 3,2 \\
Trichuris sp. + P. uncinata & 2 & 2,2 \\
E. caviae + Huevos de Capillaria $s p$. & 1 & 1,1 \\
P. uncinata + Passalurus sp. & 1 & 1,1 \\
\hline
\end{tabular}

Tabla 3. Grado de parasitismo por especie parasitaria en cuyes reproductoras, durante el periodo mayo 2016 - mayo 2019

\begin{tabular}{|c|c|c|c|c|c|c|c|c|}
\hline \multirow{3}{*}{ Especies Parasitarias } & \multicolumn{8}{|c|}{ Grado de parasitismo } \\
\hline & \multicolumn{2}{|c|}{ Negativo } & \multicolumn{2}{|c|}{ Leve } & \multicolumn{2}{|c|}{ Moderada } & \multicolumn{2}{|c|}{ Severa } \\
\hline & $\mathrm{n}$ & $\%$ & $\mathrm{n}$ & $\%$ & $\mathrm{n}$ & $\%$ & $\mathrm{n}$ & $\%$ \\
\hline P. uncinata & 199 & 79,6 & 26 & 10,4 & 25 & 10,0 &.-- &.-- \\
\hline E. caviae & 220 & 88,0 & 20 & 8,0 & 9 & 3,6 & 1 & 0,4 \\
\hline Capillaria sp. & 238 & 95,2 & 10 & 4,0 & 1 & 0,4 & 1 & 0,4 \\
\hline Balantidium sp. & 239 & 95,6 & 4 & 1,6 & 5 & 2,0 & 2 & 0,8 \\
\hline Trichuris sp. & 245 & 98,0 & 5 & 2,0 &.-- &.-- &.-- &.-- \\
\hline Passalurus sp. & 249 & 99,6 & 1 & 0,4 &.-- &.-- &.-- &.-- \\
\hline Entamoeba sp. & 249 & 99,6 & 1 & 0,4 &.-- &.-- &.-- &.-- \\
\hline
\end{tabular}


Tabla 4. Distribución de muestras positivas distribuidas por estación del año, número de partos y ambiente de crianza de cuyes reproductoras, durante el periodo mayo 2016 - mayo 2019.

\begin{tabular}{lccc}
\hline \multirow{2}{*}{ Variable } & Total de & \multicolumn{2}{c}{ Muestras positivas } \\
\cline { 3 - 4 } & Muestras & $\mathbf{n}$ & $\mathbf{\%}$ \\
\hline Estación & & & \\
Verano & 52 & 18 & $34,6^{\mathrm{a}}$ \\
Otoño & 36 & 12 & $33,3^{\mathrm{a}}$ \\
Invierno & 95 & 42 & $44,2^{\mathrm{a}}$ \\
Primavera & 67 & 21 & $31,3^{\mathrm{a}}$ \\
Número de partos & & & \\
Ninguno & 24 & 5 & $20,8^{\mathrm{a}}$ \\
I & 54 & 15 & $27,8^{\mathrm{a}}$ \\
II & 68 & 23 & $33,8^{\mathrm{a}}$ \\
III & 60 & 24 & $40,0^{\mathrm{b}}$ \\
$>$ IV & 44 & 26 & $59,1^{\mathrm{c}}$ \\
Ambiente & & & \\
Poza & 98 & 48 & $49,0^{\mathrm{a}}$ \\
Jaula & 82 & 26 & $31,7^{\mathrm{b}}$ \\
Parrilla & 70 & 19 & $27,1^{\mathrm{c}}$ \\
\hline abc Diferencia entre los grupos para cada variable evaluada &
\end{tabular}

Asimismo, el resultado está por debajo de lo publicado por Vargas et al., (2013) quien registró $76,8 \%$ en el distrito de Oxapampa; Sánchez (2013) 82,46\% en Huancayo, y García et al., (2013) 89,0\% en Ancash. Los tres estudios analizaron muestras provenientes de crianzas de tipo familiar-comercial, las que tienen un menor control en la alimentación y manejo sanitario en comparación a la crianza comercial.

Diversos estudios en nuestro medio coinciden en señalar a la nematodiasis por $P$. uncinata, como la principal parasitosis helmíntica en cuyes de producción, en ese sentido se debe mencionar que la elevada (hasta 83,0\%) (García et al., 2013; Sánchez, 2013, Suárez et al., 2014; Vargas et al., 2014; Becerra, 2015; Morales, 2017). No obstante, en el estudio P. uncinata, presenta una frecuencia baja $(20,40 \%)$, debido a las mejoras en el manejo y la alimentación que se da en los sistemas de crianza comercial, que en conjunto demuestran ser relativamente efectivos para el control de este helminto. P. uncinata, tiene una amplia distribución en América del Sur y su presencia ha sido descrita en varias familias de roedores sin mostrar una merma en su comportamiento productivo (Dean y Stephen, 2007). Sin embargo, en infestaciones altas se observan signos clínicos como pérdida de peso, debilidad y diarrea. No existen estudios que describan la patogenicidad de este helminto, la mayoría de los autores coinciden en señalar que es asintomático. Flynn y Baker (2008) consideran a $P$. uncinata como un parásito no patógeno para el cuy debido a la ausencia de lesiones en órganos. Por otro lado, Coman, Vlase, Petrut y Basescu (2009) encontraron en cortes histopatológicos de ciegos con este helminto, éctasis capilar en la submucosa, tiflitis hemorrágica y presencia de una formación nodosa localizada en el corion de la mucosa (posiblemente larva en la migración enteroparietal).

El segundo parásito encontrado con mayor frecuencia fue $E$. caviae, resultado que coincide con otros estudios de nuestro medio (Sánchez, 2013; Suárez et al., 2014; Vargas et al., 2014; Becerra, 2015). Si bien la prevalencia tiende a ser menor como es el caso de Morales (2017) que halló 9,16\%, otros reportes muestran resultados superiores al 60\% (Suárez et al., 2014; Vargas et al., 2014). La baja frecuencia podría estar relacionada al empleo de animales reproductoras que se dio en este estudio, ya que E. caviae afecta principalmente animales jóvenes durante la recría (Taylor, Coop y Wall, 2016). Otro aspecto importante fue el uso de jaulas o parrillas que minimizan la contaminación, a diferencia de los otros estudios que utilizaron pozas para la crianza lo que facilitaría el contagio entre animales. Además, actualmente en Perú los sistemas de crianza no cuentan con un patrón de desparasitación para Eimeriosis, razón por la cual 
existe todavía una exposición del parásito (Suárez et al., 2014).

E.caviae, es una coccidia específica de cuyes y puede encontrarse hasta en crianzas de tipo comercial, donde el manejo y control sanitario de los animales resulta ser más adecuado en comparación al de crianza familiar y familiar comercial. Este parásito es patógeno, siendo considerado económicamente importante, debido a la capacidad reportada de producir una rápida pérdida de peso, diarrea mucosa con estrías sanguinolentas, y/o muerte súbita en etapa de reproductores y recría, teniendo una capacidad de diseminación importante. Es posible tener un diagnóstico clínico equivocado con salmonelosis, motivo por el que la mortalidad incrementa por el uso de antibacterianos que reducen la competencia biótica bacteriana hacia las Eimerias, lo que favorece la proliferación y patogénesis propia. En la coccidiosis relacionada con E. caviae, la mortalidad está relacionada con la congestión y edema en la mucosa intestinal, con hemorragias petequiales de variada intensidad (Percy y Barthold, 2007). Debemos señalar que los animales muestreados no presentaron lesiones evidentes de enfermedad. Sin embargo, un cuadro clínico de eimeriosis puede ser potenciado por factores estresantes tales como: manejo inadecuado que sufren los cuyes en sus diferentes etapas productivas, cambios en la dieta, cambios hormonales y patrones jerárquicos, que afectan de forma negativa su sistema inmunológico (Fox, Anderson, Otto, Pritchett-Corning y Whary, 2015; Percy y Barthold, 2016).

En caso de la frecuencia encontrada para Capillaria spp., $(4,80 \%)$, los resultados son similares a las investigaciones realizadas por Dittmar (2002) 6,9\%, Sánchez (2013) 3,51\% y Becerra (2015) 6,3\%. La literatura describe a este agente como una especie no patógena, por lo que no generaría pérdidas, incluso si las cargas parasitarias fueran elevadas (Quiroz, 2005). Sin embargo, dentro de este género se encuentra Capillaria hepatica, helminto con potencial zoonótico, el que ha sido reportado en Perú afectando el hígado de cuyes silvestres (Dittmar, 2002).

Para el caso de Balantidium sp. la frecuencia reportada difiere a los resultados dados por Alves, Apolinário, Da Silva, Reis y Menezes (2007) quien reportó $78 \%$ en cuyes como animales experimentales en bioterio. Se menciona que Trichuris spp. es el helminto más frecuente en nuestro medio (Chauca, 1997), sin embargo, la frecuencia encontrada en este estudio fue baja (2.0\%) al igual que en otros estudios (Dittmar, 2002; Aquino, Chávez y Morales, 2010;
Valle y Córdova, 2012; García et al., 2013; Sánchez, 2013, Suarez et al., 2014; Vargas et al., 2014; Becerra, 2015; Morales, 2017). A pesar de ello se debe tener presente el efecto patológico al penetrar el extremo anterior del parásito en la mucosa del ciego y colon, que rompe capilares y genera diarreas mucosanguinolentas en animales jóvenes con cargas elevadas (Cordero del Campillo y Rojo, 1999; Quiroz, 2005).

Finalmente, respecto a Entamoeba sp. Sánchez (2013) reportó 3.51\% difiriendo lo hallado en la presente investigación. Ratificando, a pesar de las diferencias porcentuales, la importancia de estas especies como patógenos de los sistemas de crianza de cuyes, las cuales ocasionan infecciones que se manifiestan con anorexia, pérdida de peso, diarrea desde catarral hasta mucosa, prurito y afectación del pelaje.

En cuanto a las asociaciones de parásitos gastrointestinales con mayor frecuencia se encontró $E$. caviae + P. uncinata, siendo el primer parásito patógeno y el segundo asintomático, disminuyendo el sistema inmunológico y originando que E. cavie prolifere y ocasione mortalidad. Estudios de prevalencia de P. uncinata como el de Gárate et al., (2008) ha demostrado que este parasito es cosmopolita y de fácil adaptación a las condiciones medio ambientales. Estos resultados son similares a los encontrados por García et al. (2013) y Sánchez (2013), quienes mencionan que el monoparasitismo siempre alcanza el mayor porcentaje de frecuencia parasitaria.

En este estudio se evidenció una menor frecuencia de infestaciones moderadas y severas, estas podrían estar influenciadas por diferentes factores medioambientales relacionados a la temperatura, humedad, ventilación, entre otros; así como el tipo de manejo y factores inmunitarios. Si bien la frecuencia es baja, las infestaciones moderadas y severas producen pérdidas económicas al criador por la disminución de la producción y productividad.

En cuanto asociación entre la estación del año y la parasitosis, no se encontró asociación estadística significativa. Según reporta Vargas et al., (2014) la época del año influye directamente en la presentación de la parasitosis, la época lluviosa tiene 5.7 veces más riesgo de presentar endoparasitosis que la época seca, la menor humedad y los mayores niveles de radiación no permitirían la viabilidad de las formas infectivas de cada uno de los parásitos normalmente reportados (Urquhart, Chávez, Pinedo Morales y Suarez,2001; Barriga, 2002; Vargas et al., 2014). Asimismo, se 
encontró una asociación estadística significativa con respecto al número de partos, esto podría estar relacionado a que las hembras congregan mayor carga parasitaria conforme aumenta el número de partos.

En el caso del ambiente de crianza los animales criados en pozas presentaron mayor positividad comparados con los de crianza en jaulas y parrillas, esto debido al contaminante en la poza y la humedad lo cual genera un ambiente favorable para la multiplicación del parasito. Además, se sabe que el cuy es una especie altamente susceptible a un rango amplio de infecciones parasitarias ya que realizan la coprofagia como un mecanismo de compensación biológica. $\mathrm{Si}$ bien el material de construcción de las jaulas y la ventilación adecuada generan un ambiente favorable para la disminución de los problemas sanitarios, los resultados indican que no fue completamente eficiente en el control del parasitismo puesto que se ha obtenido resultados positivos. Por ello, merece establecer un programa sanitario calendarizado para el control de endoparásitos y otros patógenos causantes de altos índices de morbilidad y mortalidad, así como desarrollar un manejo más tecnificado y controlar factores epidemiológicos que intervienen en el desarrollo de los endoparásitos.

\section{CONCLUSIONES}

Se encontró una prevalencia de $37,2 \pm 6,0 \%$ de parásitos gastrointestinales en cuyes de crianza intensiva en Costa central.

Se registraron cuatro especies de nematodos: Paraspidodera uncinata (20.4\%), Capillaria $s p$. (4,8\%), Trichuris sp. (2,0\%) y Passalurus sp. $(0,4 \%)$; y tres especies de protozoarios: Eimeria caviae $(12,0 \%)$, Balantidium sp. (4,4\%) y Entamoeba sp. (0,4\%).

El $79,6 \%$ de las muestras correspondió a monoparasitimo y el $20,4 \%$ a biparasitismo.

El grado de parasitismo por especie parasitaria en cuyes reproductoras fueron: casos leves $26,8 \%$ $(67 / 250)$, moderados $16,0 \%(40 / 250)$ y severos $1,6 \%$ $(4 / 250)$.

Se determinó asociación estadística significativa $(\mathrm{p}<0,05)$ entre el número de partos y el ambiente de crianza frente a la parasitosis.

\section{Agradecimiento:}

El presente trabajo fue financiado por el proyecto PNIA 046-PI «Determinación de las causas de mortalidad, control de enfermedades y medidas de prevención en cuyes. Programa Nacional de Innovación Agraria (PNIA).

\section{Correspondencia}

Meylin Huamán

Correo electrónico: mhuamana@inia.gob.pe

\section{REFERENCIAS BIBLIOGRAFICAS}

1. Alves, L., Apolinário, C., Da Silva, S., Reis, S. \& Menezes, R. (2007). Endoparasitos em cobaias (Cavia porcellus) (Mammalia, Rodentia, Caviidae) provenientes de biotérios de criação e experimentação do município do Rio de Janeiro, Brasil. Ciência Rural, 37(5), 1380-1386.

2. Aquino, M., Chávez, A. \& Morales, S. (1-4 setiembre 2010). Endoparasitosis gastrointestinal en cobayos (Cavia porcellus) del distrito de San Marcos, Huaraz. En: XXII Congreso Panamericano de Ciencias Veterinarias. Lima, Perú.

3. Barriga, O. (2002). Las enfermedades parasitarias de los animales domésticos. Santiago: Ed. Germinal. $334 \mathrm{p}$.

4. Becerra, F. (2015). Frecuencia de parásitos gastrointestinales en las unidades productivas de cuyes (Cavia porcellus) de crianza intensiva en el distrito de Moquegua. (Tesis para optar el Título de Médico Veterinario y Zootecnista). Universidad Científica del Sur, Lima, Perú.

5. Beltrán, M., Tello, R., \& Náquira, C. (2003). Manual de procedimientos de laboratorio para el diagnóstico de los parásitos intestinales del hombre. Serie de Normas Técnicas $N^{\circ}$ 37. (pp. 11-12). Lima, Perú: Instituto Nacional de Salud.

6. Coman, S., Vlase, E., Petrut, T. \& Basescu, B. (2009). Aspects of the parasitary infestations of guinea pigs reared in intensive system. Revista Scentia Parasitologica, 10 (1-2), 97-100.

7. Cordero del Campillo, F. (1999). Parasitología veterinaria. Madrid: Mc Graw-Hill.968p.

8. Chauca, L. (1997). Producción de cuyes (Cavia porcellus). Roma: FAO. Recuperado de: http: //www. fao.org/docrep/w6562s/w6562s00.htm.

9. Daniel, W. (2005). Biostadistica. Base para el análisis de las Ciencias de la Salud. Ciudada de México: Editorial Limusa.

10. Dittmar, K. (2002). Arthropod and helminthes parasites of the wild guinea pig, Cavia aperea, from the Andes and the Cordillera in Peru, South America. J Parasitol, 88 (2), 409-11.

11. Dean, P. \& Stephen, B. (2007). Pathology of laboratory rodents and rabbits. 3rd ed. Iowa: Blackwell Publishing.

12. Flausino, G., Berto, B., Mcintosh, D., Furtado, 
T., Teixeira, W. \& Lopes, C.( 2014). Phenotypic and Genotypic Characterization of Eimeria caviae from Guinea Pigs (Cavia porcellus). Acta Protozool, 53 (3), 269-276.

13. Florián, A. (1999). Pérdidas de producción debido a enfermedades parasitarias. V Congreso Latinoamericano de Cuyicultura. Carcas, Venezuela.

14. Florián A. (2004). Sanidad en cuyes. Prevalencia de nematodos. Cajamarca: UTAE-INIA.

15. Flynn, R. \& Baker, D. (2008). Flynn's parasites of laboratory animals. USA: Wiley-Blackwell.

16. Fox, J.G., Anderson, L.C., Otto, G.H., PritchettCorning, K. \& Whary, M. (2015). Laboratory animal medicine. $3^{\mathrm{a}}$ ed. Londón: Elseiver.

17. Gárate, I., Cueva, B., Jiménez, P., Portilla, J., Uribe, D. \& Villar, J. (2008). Frecuencia e intensidad de infección por Paraspidodera uncinata en cobayos (Cavia sp.) sacrificados en Lima. En: XVII Reunión Científica del Instituto de Investigación de Ciencias Biológicas «Antonio Raimondi». Lima, Perú.

18. García, C., Chávez, A., Pinedo, R. \& Suárez, F. (2013). Helmintiasis gastrointestinal en cuyes (Cavia porcellus) de granjas de crianza familiar-comercial en Ancash, Perú. Rev Investig Vet Perú, 24 (4), 473-479.

19. Instituto Nacional de Investigación Agraria; Centro internacional de investigación para el desarrollo. (1991). Proyecto sistemas de producción de cuyes. Lima: INIA:

20. Instituto de Investigación Agraria. (1994). Proyecto sistemas de producción de cuyes. Lima: INIA. 99 p.

21. Layme, A., Perales, R., Chavera, A., Gavidia, C. \& Calle, S. (2011). Lesiones anatomopatológicas en cuyes (Cavia porcellus) con diagnóstico bacteriológico de salmonella sp. Rev Inv Vet Perú, 22 (4), 369-376.

22. Morales, S. (2013). La sanidad en sistema de crianza comercial de cuyes. En: XXXVI Reunión Científica Anual Asociación Peruana de Producción Animal. Cusco, Perú.

23. Morales, S. (2017). Patógenos bacterianos y parasitarios más frecuentes en cuyes de crianza familiar - comercial en tres distritos de la Provincia de Bolognesi, Departamento de Ancash en época de seca. (Tesis de Magister). Univ. Nac. Mayor de San Marcos. Lima, Perú.
24. Murga, S., Terán, M. \& Cabanillas, L. (2000), Coccidiosis intestinales en Cavia porcellus de Paiján, La libertad. En: IV congreso peruano de Parasitología. Sociedad Peruana de Parasitología. Lima, Perú.

25. Percy, D. \& Barthold, S. (2007). Pathology of Laboratory Rodents and Rabbits. Iowa: Blackwell Publishing.

26. Percy, D. \& Barthold, S. (2016). Pathology of Laboratory Rodents and Rabbits. $4^{\circ}$ ed. Edition Ames: Iowa State University Press. 206 p

27. Quiroz, H. (2005). Parasitología y enfermedades parasitarias de animales domésticos. Ciudad de Mexico: Limusa.

28. Ríos, W.H. (2018). Prevalencia de helmintiasis gastrointestinal en cuyes (Cavia porcellus) de crianza familiar-comercial en el distrito de Matahuasi, provincia de Concepción, Junín. (Tesis para optar el Título de Médico Veterinario). Universidad Nacional Mayor de San Marcos, Lima, Perú.

29. Rodríguez, V.R. \& Cob, G.L. (2005). Técnicas diagnósticas en parasitología veterinaria. $2^{\mathrm{a}}$ ed. Madrid: Universidad Autónoma de Yucatán. 299 p.

30. Sánchez, J. (2013). Estimación del parasitismo gastrointestinal en cuyes (Cavia porcellus) de la ciudad de Huancayo departamento de Junín. (Tesis para optar el Título de Médico Veterinario). Universidad Nacional Mayor de San Marcos, Lima, Perú.

31. Suárez, A., Morales, S. \& Villacaqui, E. (2014). Estudio de la parasitosis gastrointestinal en cuyes (Cavia porcellus) de crianza intensiva de la provincia de Concepción, Junín. Cientifica, 11 (1), 17-29.

32. Taylor, M.A., Coop, R.L. \& Wall R.L. (2016). Veterinary Parasitology. 3ra ed. España: Ed Blackwell Publising.

33. Urquhart, G.M., Armour, J., Duncan, J.L., Dunn, A.M. \& Jennings, F.W. (2001). Parasitología veterinaria. 2da ed. Zaragoza: Ed. Acribia.

34. Vargas, M., Chávez, A., Pinedo, R., Morales, S. \& Suarez, F. (2014). Parasitismo gastrointestinal en dos épocas del año en cuyes (Cavia porcellus) de Oxapampa, Pasco. Rev Inv Vet Perú, 25 (2), 276-283.

35. Valle, M. \& Córdova, L. (2012). Estudio de parasitosis en cuyes de la corporación agroproductiva del Cantón Ambato, su influencia en parámetros productivos y establecimientos de programas de bioseguridad especifica. (Tesis de Grado). Universidad Estatal de Bolívar, Guaranda, Ecuador. 